\title{
A Generlization of the Eneström-Kakeya Theorem
}

Pukhta MS*

Division of Agriculture Statistics, Sher-e-Kashmir, University of Agricultural Sciences and Technology of Kashmir-191101, India

\begin{abstract}
In this paper we obtain a generalization of well-known result of Eneström -Kakeya concerning the bounds for the moduli of the zeros of polynomials with complex coefficients which improve upon some results due to A. Aziz and Q.G Mohammad and others.
\end{abstract}

Keywords: Polynomial; Zeros; Eneström-Kakeya Theorem

\section{Introduction and Statement of Results}

The following result known as Eneström - Kakeya theorem, is well known in the theory of distribution of zeros of polynomials was firstly proved by Eneström [1] and Kakeya [2].

Theorem 1.1: If $p(z)=\sum_{j=0}^{n} a_{j} z^{j}$ is a polynomial of degree $\mathrm{n}$ with real coefficients such that

$$
a_{n} \geq a_{n-1} \geq \ldots \geq a_{1} \geq a_{0}>0,
$$

then all the zeros of $p(z)$ lie in $|z| \leq 1$.

In literature [3-5] there exist several extensions of Eneström -Kakeya theorem. By using Schwartz lemma, Aziz and Mohammad [6] generalized Eneström -Kakeya theorem in a different way and proved:

Theorem 1.2: If $p(z)=\sum_{j=0}^{n} a_{j} z^{j}$ be a polynomial of degree $\mathrm{n}$ with real positive coefficients. If $t_{1}^{j=0}>t_{2} \geq 0$ can be found such that

$a_{r} t_{1} t_{2}+a_{r-1}\left(t_{1}-t_{2}\right)-a_{r-2} \geq 0, r=1,2, \ldots, n+1 ; a_{-1}=a_{n+1}=0$,

then all the zeros of $p(z)$ lie in $|z| \leq t_{1}$.

The following generalization of Theorem $B$ is due to Rather et al [7].

Theorem 1.3: If $P(z)=\sum_{j=0}^{n} a_{j} z^{j}$ be a polynomial of degree $\mathrm{n}$ with $\operatorname{Re} \alpha_{j}=\alpha_{j}$ and

$\operatorname{Im} \alpha_{j}=\beta_{j}, j=0,1,2, \ldots \ldots, n$ If $t_{1}>t_{2} \geq 0$, can be found such that

$t_{1} t_{2} \alpha_{r}+\left(t_{1}-t_{2}\right) \alpha_{r-1}-\alpha_{r-2} \geq 0$ for $r=1,2,3, \ldots \ldots, k+1$,

$t_{1} t_{2} \alpha_{r}+\left(t_{1}-t_{2}\right) \alpha_{r-1}-\alpha_{r-2} \leq 0$ for $r=k+2, \ldots \ldots, n+1$,

and

$t_{1} t_{2} \beta_{r}+\left(t_{1}-t_{2}\right) \beta_{r-1}-\beta_{r-2} \geq 0$ for $r=1,2,3, \ldots . ., m+1$,

$t_{1} t_{2} \beta_{r}+\left(t_{1}-t_{2}\right) \beta_{r-1}-\beta_{r-2} \leq 0$ for $r=m+2, \ldots \ldots, n+1$,

$0 \leq k \leq n, 0 \leq m \leq n, \alpha_{-1}=\beta_{-1}=\alpha_{n+1}=\beta_{n+1}=0, \alpha_{n}>0$ then all the zeros of $P(z)$ lie in

$$
|z| \leq \frac{t_{1}}{\left|a_{n}\right|}\left\{2 t_{1}^{k-n}\left(\alpha_{k}+t_{2} \alpha_{k+1}\right)+2 t_{1}^{m-n}\left(\beta_{m}+t_{2} \beta_{m+1}\right)-\left(\alpha_{n}+\beta_{n}\right)\right\}
$$

In this paper, as a generalization of Theorem (1.3), We prove the following result.

Theorem1.4: Let $P(z)=\sum_{j=0}^{n} a_{j} z^{j}$ be a polynomial of degree $n \geq 1$ with $\operatorname{Re} \alpha_{j}=\alpha_{j}$ and $\operatorname{Im} \alpha_{j}=\beta_{j}, j=0,1,2, \ldots \ldots ., n$. If $t_{1} \geq t_{2} \geq 0, t_{1} \neq 0$ can be found such that

$$
t_{1} t_{2} \alpha_{r}+\left(t_{1}-t_{2}\right) \alpha_{r-1}-\alpha_{r-2} \geq 0 \text { for } r=1,2,3, \ldots ., k+1,
$$

$$
t_{1} t_{2} \alpha_{r}+\left(t_{1}-t_{2}\right) \alpha_{r-1}-\alpha_{r-2} \leq 0 \text { for } r=k+2, \ldots \ldots, n+1
$$

and

$$
\begin{aligned}
& t_{1} t_{2} \beta_{r}+\left(t_{1}-t_{2}\right) \beta_{r-1}-\beta_{r-2} \geq 0 \text { for } r=1,2,3, \ldots \ldots, m+1, \\
& t_{1} t_{2} \beta_{r}+\left(t_{1}-t_{2}\right) \beta_{r-1}-\beta_{r-2} \leq 0 \text { for } r=m+2, \ldots \ldots, n+1, \\
& 0 \leq k \leq n-1,0 \leq m-1 \leq n, \alpha_{-1}=\beta_{-1}=\alpha_{n+1}=\beta_{n+1}=0, \alpha_{n}>0 \text { then all the }
\end{aligned}
$$
zeros of $\mathrm{P}(\mathrm{z})$ lie in

$$
\begin{aligned}
& \left|z+\frac{\alpha_{n-1}-\left(t_{1}-t_{2}\right) \alpha_{n}}{a_{n}}\right| \leq 2\left(\alpha_{k+1} t_{2}+\alpha_{k}\right) \frac{t_{1}^{k+1}}{\left|a_{n}\right| t_{1}^{n}}+2\left(\beta_{m+1} t_{2}+\beta_{m}\right) \frac{t_{1}^{m+1}}{\left|a_{n}\right| t_{1}^{n}} \\
& -\frac{t_{2} \alpha_{n}+t_{1} \beta_{n}+\alpha_{n-1}}{\left|a_{n}\right|}
\end{aligned}
$$

For $\beta_{j}=0, j=0,1,2, \ldots, n$ in the Theorem (1.4), we obtain the following result.

Corollary 1.5: Let $P(z)=\sum_{j=0}^{n} a_{j} z^{j}$ be a polynomial of degree $n \geq 3$ with real and positive coefficients. If $t_{1} \geq t_{2} \geq 0, t_{1} \neq 0$ can be found such that

$$
\begin{aligned}
& t_{1} t_{2} \alpha_{r}+\left(t_{1}-t_{2}\right) \alpha_{r-1}-\alpha_{r-2} \geq 0 \text { for } r=1,2,3, \ldots ., k+1, \\
& t_{1} t_{2} \alpha_{r}+\left(t_{1}-t_{2}\right) \alpha_{r-1}-\alpha_{r-2} \leq 0 \text { for } r=k+2, \ldots \ldots, n+1, \\
& 0 \leq k \leq n-1 a_{-1}=a_{n+1}=0, \text { then all the zeros of } \mathrm{P}(\mathrm{z}) \text { lie in } \\
& \left|z+\frac{a_{n-1}}{a_{n}}-\left(t_{1}-t_{2}\right)\right| \leq t_{2}+\frac{a_{n-1}}{a_{n}}+\frac{2 t_{2} \alpha_{k+1}+2 \alpha_{k}}{a_{n} t_{1}^{n-k+1}}
\end{aligned}
$$

\section{Remark}

In general Theorem 1.4 also gives much better result than Theorem 1.3 for $0 \leq k \leq n-1$. For this we show that the circle defined by (1.5) is contained in the circle defined by (1.3). Let $\mathrm{z}=\mathrm{w}$ be any point belonging to the circle defined by (1.4) then

${ }^{*}$ Corresponding author: Pukhta MS, Division of Agriculture Statistics, Sher-eKashmir, University of Agricultural Sciences and Technology of Kashmir-191101, India, Tel: 01954262260; E-mail: mspukhta_67@yahoo.co.in

Received January 08, 2014; Accepted May 29, 2014; Published June 02, 2014

Citation: Pukhta MS (2014) A Generlization of the Eneström-Kakeya Theorem. J Appl Computat Math 3: 164 doi:10.4172/2168-9679.1000164

Copyright: (c) 2014 Pukhta MS. This is an open-access article distributed under the terms of the Creative Commons Attribution License, which permits unrestricted use, distribution, and reproduction in any medium, provided the original author and source are credited. 


$$
\begin{aligned}
& \left|w+\frac{\alpha_{n-1}-\left(t_{1}-t_{2}\right) \alpha_{n} \mid}{a_{n}}\right| \leq 2\left(\alpha_{k+1} t_{2}+\alpha_{k}\right) \frac{t_{1}^{k+1}}{\left|a_{n}\right| t_{1}^{n}}+2\left(\beta_{m+1} t_{2}+\beta_{m}\right) \frac{t_{1}^{m+1}}{\left|a_{n}\right| t_{1}^{n}} \frac{t_{2} \alpha_{n}+t_{1} \beta_{n}+\alpha_{n-1}}{\left|a_{n}\right|} \text { This gives } \\
& |w|=\left|w+\frac{\alpha_{n-1}-\left(t_{1}-t_{2}\right) \alpha_{n}}{a_{n}}-\frac{\alpha_{n-1}-\left(t_{1}-t_{2}\right) \alpha_{n}}{a_{n}}\right| \\
& \leq\left|w+\frac{\alpha_{n-1}-\left(t_{1}-t_{2}\right) \alpha_{n}}{a_{n}}\right|+\left|\frac{\alpha_{n-1}-\left(t_{1}-t_{2}\right) \alpha_{n}}{a_{n}}\right| \\
& \leq 2\left(\alpha_{k+1} t_{2}+\alpha_{k}\right) \frac{t_{1}^{k+1}}{\left|a_{n}\right| t_{1}^{n}}+2\left(\beta_{m+1} t_{2}+\beta_{m}\right) \frac{t_{1}^{m+1}}{\left|a_{n}\right| t_{1}^{n}}-\frac{t_{2} \alpha_{n}+t_{1} \beta_{n}+\alpha_{n-1}}{\left|a_{n}\right|}+\frac{\alpha_{n-1}-\left(t_{1}-t_{2}\right) \alpha_{n}}{\left|a_{n}\right|} \\
& =\frac{t_{1}}{\left|a_{n}\right|}\left\{2 t_{1}^{k-n}\left(\alpha_{k}+t_{2} \alpha_{k+1}\right)+2 t_{1}^{m-n}\left(\beta_{m}+t_{2} \beta_{m+1}\right)-\left(\alpha_{n}+\beta_{n}\right)\right\}
\end{aligned}
$$

Hence the point $\mathrm{z}=\mathrm{w}$ belongs to the circle defined by (1.3) and therefore, the circle defined by (1.4) is contained in the circle (1.3).

\section{Proof of the Theorem}

Proof of Theorem 1.4: Consider the polynomial

$$
\begin{aligned}
G(z)= & \left(t_{1}-z\right)\left(t_{2}+z\right) P(z) \\
= & -a_{n} z^{n+2}+\left\{a_{n}\left(t_{1}-t_{2}\right)-a_{n-1}\right\} z^{n+1} \\
& +\sum_{v=2}^{n}\left\{a_{v} t_{1} t_{2}+a_{v-1}\left(t_{1}-t_{2}\right)-a_{v-2}\right\} z^{v} \\
& +\left\{a_{1} t_{1} t_{2}+a_{0}\left(t_{1}-t_{2}\right)\right\} z+a_{0} t_{1} t_{2} \\
= & -a_{n} z^{n+2}+\left\{a_{n}\left(t_{1}-t_{2}\right)-a_{n-1}\right\} z^{n+1} \\
& +\sum_{v=2}^{n}\left\{a_{v} t_{1} t_{2}+a_{v-1}\left(t_{1}-t_{2}\right)-a_{v-2}\right\} z^{v} \quad\left(a_{-2}=a_{-1}=0\right)
\end{aligned}
$$

Let $|z|>t_{1}$, then

$$
\begin{aligned}
& |G(z)| \geq|z|^{n+1}\left\{\left|a_{n} z+a_{n-1}-\left(t_{1}-t_{2}\right) a_{n}\right|-\sum_{v=0}^{n}\left|a_{v} t_{1} t_{2}+a_{v-1}\left(t_{1}-t_{2}\right)-a_{v-2}\right| \frac{1}{|z|^{n-v+1}}\right\} \\
& >|z|^{n+1}\left\{\left|a_{n} z+\alpha_{n-1}-\left(t_{1}-t_{2}\right) \alpha_{n}\right|-\left|\beta_{n-1}\right|-\left(t_{1}-t_{2}\right)\left|\beta_{n}\right|-\sum_{v=0}^{n}\left|a_{v} t_{1} t_{2}+a_{v-1}\left(t_{1}-t_{2}\right)-a_{v-2}\right| \frac{1}{t_{1}^{n-v+1}}\right\}
\end{aligned}
$$

Now by hypothesis

$\sum_{v=0}^{n}\left|a_{v 1} t_{1} t_{2}+a_{v-1}\left(t_{1}-t_{2}\right)-a_{v-2}\right| t_{1}^{v} \leq\left.\sum_{v=0}^{n}\left|\alpha_{v} t_{1} t_{2}+\alpha_{v-1}\left(t_{1}-t_{2}\right)-\alpha_{v-2}\right|\right|_{1} ^{v}+\sum_{v=0}^{n}\left|\beta_{v} t_{1} t_{2}+\beta_{v-1}\left(t_{1}-t_{2}\right)-\beta_{v-2}\right| t_{1}^{v}$

$$
\begin{aligned}
& \leq \sum_{v=0}^{k+1}\left|\alpha_{v} t_{1} t_{2}+\alpha_{v-1}\left(t_{1}-t_{2}\right)-\alpha_{v-2}\right| t_{1}^{v} \\
& \sum_{v=k+2}^{n}\left|\alpha_{v} t_{1} t_{2}+\alpha_{v-1}\left(t_{1}-t_{2}\right)-\alpha_{v-2}\right| t_{1}^{v} \\
& +\sum_{v=0}^{m+1}\left|\beta_{v} t_{1} t_{2}+\beta_{v-1}\left(t_{1}-t_{2}\right)-\beta_{v-2}\right| t_{1}^{v} \\
& +\sum_{v=m+1}^{n}\left|\beta_{v} t_{1} t_{2}+\beta_{v-1}\left(t_{1}-t_{2}\right)-\beta_{v-2}\right| t_{1}^{v} \\
& =2\left(\alpha_{k+1} t_{2}+\alpha_{k}\right) t_{1}^{k+2}-\left(\alpha_{n} t_{2}+\alpha_{n-1}\right) t_{1}^{n+1} \\
& +2\left(\beta_{m+1} t_{2}+\beta_{m}\right) t_{1}^{m+2}-\left(\beta_{n} t_{2}+\beta_{n-1}\right) t_{1}^{n+1}
\end{aligned}
$$

Using this in (1.6), we obtain

$$
\begin{aligned}
& |G(z)| \geq|z|^{n+1} \\
& \left\{\begin{array}{l}
\left|a_{n} z+\alpha_{n-1}-\left(t_{1}-t_{2}\right) \alpha_{n}\right| \\
\left.-\left(\beta_{n-1}-\left(t_{1}-t_{2}\right) \beta_{n}\right)-2\left(\alpha_{k+1} t_{2}+\alpha_{k}\right) \frac{1}{t_{1}^{n-k-1}}+\left(\alpha_{n} t_{2}+\alpha_{n-1}\right)-2\left(\beta_{m+1} t_{2}+\beta_{m}\right)\right\} \\
\frac{1}{t_{1}^{n-m-1}}+\left(\beta_{n} t_{2}+\beta_{n-1}\right)
\end{array}\right. \\
= & \left.\left.\begin{array}{l}
|z|^{n+1} \\
\left\{a_{n} z+\alpha_{n-1}-\left(t_{1}-t_{2}\right) \alpha_{n} \mid+t_{1} \beta_{n}-2\left(\alpha_{k+1} t_{2}+\alpha_{k}\right)\right.
\end{array}\right\} \frac{1}{t_{1}^{n-k-1}}+\left(\alpha_{n} t_{2}+\alpha_{n-1}\right)-2\left(\beta_{m+1} t_{2}+\beta_{m}\right) \frac{1}{t_{1}^{n-m-1}}\right\} \\
\quad & \quad-\left(t_{2} \alpha_{n}+t_{1} \beta_{n}+\alpha_{n-1}\right)
\end{aligned}
$$

Hence all the zeros of $G(z)$ whose modulus is greater than $t_{1}$ lie in the circle

$$
\begin{aligned}
& \left|z+\frac{\alpha_{n-1}-\left(t_{1}-t_{2}\right) \alpha_{n}}{a_{n}}\right| \leq 2\left(\alpha_{k+1} t_{2}+\alpha_{k}\right) \frac{t_{1}^{k+1}}{\left|a_{n}\right| t_{1}^{n}}+2\left(\beta_{m+1} t_{2}+\beta_{m}\right) \frac{t_{1}^{m+1}}{\left|a_{n}\right| t_{1}^{n}} \\
& -\frac{t_{2} \alpha_{n}+t_{1} \beta_{n}+\alpha_{n-1}}{\left|a_{n}\right|}
\end{aligned}
$$

Now we show that all the zeros of $G(z)$ whose modulus is less than equal to $t_{1}$ also lie in the circle defined by (1.4). Let $|z| \leq t_{1}$, then we have

$$
\begin{aligned}
& \left|a_{n} z+\alpha_{n-1}-\left(t_{1}-t_{2}\right) \alpha_{n}\right| \leq\left|a_{n}\right| t_{1}+\left|\alpha_{n-1}-\left(t_{1}-t_{2}\right) \alpha_{n}\right| \\
& \leq t_{1} \alpha_{n}+t_{1} \beta_{n}+\alpha_{n-1}-\left(t_{1}-t_{2}\right) \alpha_{n} \\
& =2 t_{1} \beta_{n}+2\left(t_{2} \alpha_{n}+\alpha_{n-1}\right)
\end{aligned}
$$

By hypothesis

$$
\sum_{v=k+2}^{n} \frac{a_{v} t_{1} t_{2}+a_{v-1}\left(t_{1}-t_{2}\right)-a_{v-2}}{t_{1}^{n-v+1}} \leq 0,0 \leq k \leq n-1
$$

This gives

$$
2\left(t_{2} \alpha_{n}+\alpha_{n-1}\right) \leq \frac{2\left(\alpha_{k+1} t_{2}+\alpha_{k}\right)}{t_{1}^{n-k-1}}
$$

Similarly for $0 \leq m \leq n-1$

$$
2\left(t_{2} \beta_{n}+\beta_{n-1}\right) \leq \frac{2\left(\beta_{m+1} t_{2}+\beta_{m}\right)}{t_{1}^{n-m-1}}
$$

Also we have

$t_{1} \beta_{n} \leq t_{2} \beta_{n}+\beta_{n-1}$

Combining (1.9) and (1.10), we obtain

$2 t_{1} \beta_{n} \leq \frac{2\left(\beta_{m+1} t_{2}+\beta_{m}\right)}{t_{1}^{n-m-1}}$

Using (1.8) and (1.11) in (1.7), we have

$$
\begin{gathered}
\left|a_{n} z+\alpha_{n-1}-\left(t_{1}-t_{2}\right) \alpha_{n}\right| \leq 2\left(\alpha_{k+1} t_{2}+\alpha_{k}\right) \frac{t_{1}^{k+1}}{\left|a_{n}\right| t_{1}^{n}}+2\left(\beta_{m+1} t_{2}+\beta_{m}\right) \frac{t_{1}^{m+1}}{\left|a_{n}\right| t_{1}^{n}} \\
-\left(t_{2} \alpha_{n}+t_{1} \beta_{n}+\alpha_{n-1}\right) .
\end{gathered}
$$


Since all the zeros of $P(z)$ are also the zeros of $G(z)$, we conclude that all the zeros of $P(z)$ lie in

$$
\begin{aligned}
& \left|z+\frac{\alpha_{n-1}-\left(t_{1}-t_{2}\right) \alpha_{n}}{a_{n}}\right| \leq 2\left(\alpha_{k+1} t_{2}+\alpha_{k}\right) \frac{t_{1}^{k+1}}{\left|a_{n}\right| t_{1}^{n}}+2\left(\beta_{m+1} t_{2}+\beta_{m}\right) \frac{t_{1}^{m+1}}{\left|a_{n}\right| t_{1}^{n}} \\
& -\frac{t_{2} \alpha_{n}+t_{1} \beta_{n}+\alpha_{n-1}}{\left|a_{n}\right|}
\end{aligned}
$$

This completes the proof of the theorem.

\section{References}

1. Eneström G (1920) Ramarque sur un theorem relative aux recines de l'equation $a_{n} z^{n}+$ $+a_{0}=0$ ou tous les coefficients sont reels et positives, Tohoku Math J 18: 36-40
2. Kakeya $S$, On the limits of the roots of an algebraic equation with positive coefficients. Tohoku Math 140-142.

3. Anderson N, Saff EB, Verga RS (1979) On the Eneström -Kakeya theorem and its sharpness. Linear Algebra and Applications 28: 5-16.

4. Anderson N, Saff EB, Verga RS (1981) An extension of the Eneström -Kakeya theorem and its sharpness, SIAM J Math Anal 12: 10-22.

5. Cargo GT, Shishla O (1963) Zeros of polynomials and fractional order differences of their Coefficients. J Math Anal Appl 7: 176-182.

6. Aziz A, Mohammad QG (1984) Zero free regions for polynomials and some generalizations of Eneström-Kakeya theorem, Canad Math Bull 2: 265- 272.

7. Rather NA, Shakeel A, Simnani M I Mir (2007) On the Eneström-Kakeya theorem, Intl J Pure and Appl Math 41: 807-815. 